\title{
HIPERFRECUENTACIÓN MÉDICA EN MAYORES Y SALUD
}

\author{
José Manuel Aguilar-Parra \\ jmaguilar@ual.es \\ Remedios López Liria \\ rll040@ual.es \\ David Padilla Góngora \\ dpadilla@ual.es \\ Patricia Rocamora Pérez \\ rocamora@ual.es \\ Ana Morales Montoya \\ moralesmontoyaana@gmail.com, \\ Universidad de Almería
}

Fecha de Recepción: 18 Febrero 2018

Fecha de Admisión: 10 Abril 2018

\section{RESUMEN}

El envejecimiento demográfico de la población, su intensificación a medio plazo y el incremento de los gastos farmacológicos, sanitarios y en servicios sociales de las personas mayores pone en riesgo la sostenibilidad económica del presupuesto sociosanitario público, por lo que es necesario mejorar la eficacia de este gasto. El objetivo de este estudio es realizar una revisión del estado de la cuestión sobre el uso excesivo, por parte de algunas personas mayores, de los servicios sanitarios. De forma que se defina cuál es el perfil de ese mayor y los hábitos que lo caracterizan. Se ha realizado una revisión sistemática en las bases de datos Scopus, Pubmed y Web of Science utilizando como descriptores: hiperfrecuentación médica (number of visits to the doctor), gasto en salud (heath expenditure), personas mayores (elderly) y calidad de vida (quality of life). Según los estudios consultados un mayor de 65 años gasta 3,2 veces más en salud que personas de menor edad, consumiendo el 40,3 del gasto total en sanidad. Desde el enfoque subjetivo, se centra en la percepción personal de la salud, un aspecto que es esencial a la hora de predecir o buscar ayuda médica. De hecho, la percepción del propio estado de salud está más asociada al uso de los servicios de salud que la propia condición clínica o patológica del sujeto. Otro de los problemas más relevantes en la indagación del estado de salud es la importancia que tiene el autoconcepto y la autoestima, junto con el estado de ánimo del sujeto, en la evaluación que hace el mismo de su estado de salud. Tanto una autoestima baja como los estados y procesos depresivos tienden a ir asociados con una evaluación negativa de la misma y todo ello, a requerir un mayor uso de los servicios sanitarios, sin existir, en muchas ocasiones una necesidad real de atención médica. 


\section{HIPERFRECUENTACIÓN MÉDICA EN MAYORES Y SALUD}

Palabras clave: hiperfrecuentación médica; personas mayores; calidad de vida; salud; gasto en salud

\section{ABSTRACT}

Medical hyperhealthresource in older and health.

The demographic aging of the population, its intensification in the medium term and the increase in pharmacological, health and social services expenses of the elderly puts the economic sustainability of the public health budget at risk, which is why it is necessary to improve the effectiveness of this spending. The aim of this study is to conduct a review of the state of the question about the excessive use, by some elderly people, of health services. In a way that defines the profile of that elder and the habits that characterize it. A systematic review has been carried out in the Scopus, Pubmed and Web of Science databases using as descriptors: medical frequent attenders, health expenditure, elderly people and quality of life. According to the studies consulted, a person older than 65 years spends 3.2 times more in health than younger people, consuming 40.3 of the total health expenditure. From the subjective approach, it focuses on the personal perception of health, an aspect that is essential when it comes to predicting or seeking medical help. In fact, the perception of one's health condition is more associated with the use of health services than the subject's own clinical or pathological condition. Another of the most relevant problems in the investigation of the state of health is the importance of self-concept and self-esteem, together with the state of mind of the subject, in the evaluation made by the same of his health status. Both a low self-esteem and states and depressive processes tend to be associated with a negative evaluation of the same and all this, to require greater use of health services, without there being, in many cases, a real need for medical attention.

Keywords: frequent attenders; elderly; quality of life; health; health expenditure

\section{ANTECEDENTES}

Numerosos estudios han encontrado que una parte importante de las consultas (30-40\%) en los servicios de atención primaria (AP) las realizan una pequeña proporción de pacientes (5-10\%)1-3. Estos pacientes, denominados hiperfrecuentadores (en inglés frequent attenders o high utilizers) suponen una considerable carga de trabajo (workload) para la medicina de familia, además de un alto coste económico para el sistema sanitario público (Luciano y Serrano, 2008).

Tal y como señalan Rodríguez, Cebrià, Corbella, Segura, y Sobrequés (2003), los pacientes hiperfrecuentadores son motivo de preocupación para el sistema sanitario ya que ocupan más del $50 \%$ del tiempo de consulta del médico. Además, significa que son unos pocos usuarios los que generan la mayoría de trabajo. La hiperfrecuentación engloba aspectos de tipo biológico, psicológico y social. Se ha descrito que un $50 \%$ del personal médico de atención primaria considera que recibe más del 20 \% de visitas innecesarias (Sihvonen, y Kekki, 1990). En este sentido, Arillo et al., (2006) y Turabian et al., (1988), citados en Sandín-Vázquez, y Conde-Espejo (2010), aclaran que la hiperfrecuentación es uno de los aspectos más complejos y preocupantes de la práctica diaria en la atención primaria (AP) ya que, unido a la falta de tiempo para la consulta y la consecuente masificación, afecta a la calidad del servicio prestado en la consulta. Se ha descrito que un $50 \%$ del personal médico de AP considera que más del $20 \%$ de las visitas que reciben serían innecesarias, y este fenómeno ocasiona emociones de difícil manejo entre los profesionales.

Guerra y Anca (2007) señalan que en atención primaria (AP) en particular se observan incrementos del $17 \%$ para un mismo número de habitantes con problemas de coste, demoras en la cobertura de servicios, desencuentro entre oferta-expectativas, dudas sobre coste-efectividad, equidad, calidad de los servicios y alta presión asistencial (la de España es de las más altas de Europa). 
Los pacientes hiperfrecuentadores suponen un gasto considerable para el sistema público de salud. De hecho, entre un $5 \%$ y un $10 \%$ de las consultas a un médico de atención primaria (porcentaje de hiperfrecuentadores con respecto al total de atenciones del médico) consume un $30 \%$ de esfuerzo y tiempo de él. Hay que añadir además el gasto de las pruebas complementarias ordenadas que nunca llegan a ser concluyentes a la hora de diagnosticar trastorno físico alguno (Robles, 2008, citado en Giraldo, Rincón, Uribe, y Cardona, 2010).

"El paciente que acude con gran frecuencia a la consulta no tiene por qué ser un problema, pero a menudo lo es. La mayoría de ellos presentan multitud de motivos de consulta, más o menos cambiantes, frecuentemente de poca importancia, aunque a veces vienen motivados por problemas crónicos e incapacitantes. Hay gente a la que le gusta acudir a la consulta. Por el profesional, por el tiempo que ocupan, por la relación que establecen con otros pacientes, etc. Otras veces refleja la intolerancia y falta de aceptación ante distintos padecimientos crónicos" (Giraldo, Rincón, Uribe, y Cardona, 2010).

La hiperfrecuentación se computa de diferentes maneras, atendiendo diferentes estudios. En la literatura científica no existe una definición universal y unánime de paciente hiperfrecuentador (HF) y cualquier punto de corte resulta arbitrario. No obstante, a continuación se recoge una revisión de lo subscrito en diferentes estudios.

Normalmente, en muchos de los estudios, se evalúa mediante el número de visitas registradas en la base de datos del centro. Se consideran pacientes hiperfrecuentadores a aquellos que consultaron más de 10 veces al médico de familia el año anterior por iniciativa propia; es decir, 10 visitas espontáneas; descartando aquellas visitas programadas por el médico ni derivadas de enfermería (Bellón, 1995). Como también muchos estudios consideran otros criterios para la inclusión de pacientes en estudios de hiperfrecuentación, más de 14 veces al año (Rivera-Casares, et al., 2000).

Sarría, Timoner, y Sandín (2005) señalan que se aplican diferentes criterios cuantitativos a la hora de investigar el problema y sus posibles causas (número de visitas al año, pacientes que superan el percentil 950 el percentil 90 de visitas, pacientes que superan la media $+2 \mathrm{DE}$ ).

Los hiperfrecuentadores (definidos en algunos estudios como > 14 años y con más de 8 visitas/médico/año y en otros por un número de visitas superior a 2 desviaciones estándar de la media de frecuentación) 15 son una parte importante de la demanda, ya que suponen el $12-21 \%$ de los pacientes de AP y causan el 46-50\% de las consultas (Neal, Heywood, Morley, Clayden, y Dowell, 1998, Reid, Crayford, Patel,Wessely, y Hotopf, 2003, y Ortega, Roca, Iglesias, Jurado, y Ortega, 2004, citados en Guerra y Anca, 2007).

En colombia, Giraldo, Rincón, Uribe, y Cardona (2010) señalan que los pacientes que consultan repetidamente al médico, reciben el nombre de "hiperfrecuentador", según la literatura científica internacional, cuando consultan siete 0 más veces en el año. Los pacientes hiperfrecuentadores no siempre son aquellos que hacen inadecuado uso de los servicios de salud, especialmente de la consulta médica. Hay pacientes que consultan varias veces por su condición patológica crónica y no son considerados hiperfrecuentadores, como es el caso de los usuarios pertenecientes a programas de promoción y prevención (hipertensos, diabéticos, etc.)

En un estudio realizado por Luciano y Serrano (2008), apuntan que para clasificar al paciente como hiperfrecuentador, que se tendrían que tener en cuenta criterios estadísticos y clínicos, y consistiría básicamente en agrupar a los pacientes que consultan en AP en 4 categorías clínicas: a) sin enfermedad física o mental crónica; b) con diagnóstico de enfermedad física crónica; c) con diagnóstico de trastorno mental (siguiendo criterios DSM-IV o CIE-10), y d) con comorbilidad física y mental crónica. Una vez clasificados los pacientes, se definiría como hiperfrecuentadores dentro de cada categoría a todos aquellos que se encontrasen por encima del percentil 75 en el número anual 


\section{HIPERFRECUENTACIÓN MÉDICA EN MAYORES Y SALUD}

de visitas. Como consecuencia, para ser considerados hiperfrecuentadores, los pacientes sin enfermedades, con trastorno mental, con enfermedades físicas crónicas o con comorbilidad física-psíquica, tendrían que haber realizado, respectivamente, más de 4, 6, 7 y 10 visitas a su médico de familia en el último año. Sería recomendable que futuras investigaciones analizaran la sobreutilización de los servicios en AP por categorías clínicas como las propuestas y su correspondiente percentil 75 (o percentil 90 si se desea detectar a los extremadamente frecuentadores).

En unos casos se utiliza un punto de corte en la distribución de la frecuentación, siendo los más utilizados los percentiles 75 o 90. En otros estudios se define hiperfrecuentación según el número de consultas en un periodo de tiempo determinado, la mayor parte de las veces entre 9 y 14 consultas en un año (Gill, y Sharp, 1999; Luciano et al., 2010; Vedsted, y Christensen, 2015).

Algunos autores sienten la necesidad de redefinir este concepto y prefieren hablar de sobreutilización cuando se produce por encima de la que se precisa para el perfil de riesgo del paciente (Sarría, Timoner y Sandín, 2005).

Siguiendo varios estudios, se puede ver como las personas más mayores son más hiperfrecuentadores (Fuertes et al., 1994; Sandín y, Chorot, 1990). También que las mujeres son mayores hiperfrecuentadoras que los hombres (Llorente et al., 1996; Rubio, et al., 1998).

A estos pacientes con un alto uso de los servicios sanitarios y con alto consumo farmacológico se les consideran pacientes vulnerables (Alpern, et al., 2014; Cotonat, Bertolet, Ocaña, y Gatius, 2014).

\section{OBJETIVO}

El objetivo de este estudio es realizar una revisión del estado de la cuestión sobre el uso excesivo, por parte de algunas personas mayores, de los servicios sanitarios. De forma que se defina cuál es el perfil de ese mayor y los hábitos que lo caracterizan.

\section{METODOLOGÍA}

Se ha realizado una revisión sistemática en las bases de datos Pubmed, Scopus y Web of Science utilizando como descriptores: hiperfrecuentación médica (number of visits to the doctor / frequent attenders), gasto en salud (heath expenditure), personas mayores (elderly) y calidad de vida (quality of life).

\section{RESULTADOS}

Tal y como describen Giraldo, Rincón, Uribe, y Cardona (2010), ha habido diversas investigaciones centradas en el estudio de las características sociodemográficas y de utilización de servicios más comunes entre las personas hiperfrecuentadoras. Ellas han hallado que un gran porcentaje de éstas padece alguna enfermedad crónica (Dowrick, Bellón, y Gómez, 2010), elevados índices de estrés de vida (Baez, Aiarzaguena, Grandes, Pedrero, Aranguren, y Retolaza, 1998) y que los síntomas depresivos constituyen el mejor predictor de la hiperfrecuentación (Ortega, Roca, Iglesias, y Jurado, 2004). Se han realizado intervenciones con estos pacientes, con los cuales se consiguió reducir la utilización de los servicios y se determinaron las posibles causas de la hiperfrecuentación (Bellón, Rodríguez, Castillo, Gallardo, Caballero de las Olivas, Moreno, 2004). Vidal et al., (2010) señalan que los pacientes hiperfrecuentadores tienen una percepción de menor apoyo social del grupo primario (familia) respecto al grupo normofrecuentador, pudiendo ser este apoyo el que ofreciera un marco de contención en el paciente y por tanto, el tipo de apoyo relacionado con el patrón de frecuentación en las consultas de Atención Primaria. Estos mismos autores señalaron que la mayoría declaró acudir al médico de familia por malestar físico, aunque un porcentaje nada desdeñable afirmó acudir por motivos tanto físicos como psicológicos (alrededor del 25\%), además, se 
observó una media de visitas anuales de 26 ( $D S=4,2)$. Esto supone que el grupo de hiperfrecuentadores acude a visitarse a las consultas de atención primaria una proporción de 4 veces más que la media de normofrecuentadores.

En un estudio similar, Aguilar-Palacio, Carrera-Lasfuente, Solsona, Sartolo y Rabanaque (2016) apuntaban que las mujeres trabajadoras manuales presentaron la mayor prevalencia de mala salud (mala salud percibida 70,6\%). La mala salud se asoció a mayor utilización de servicios sanitarios. La salud percibida fue mejor predictor de utilización de servicios y de hiperfrecuentación que la diagnosticada, con la mayor capacidad predictiva para $\operatorname{AE}(C=0,676)$. Los ancianos de clases sociales bajas utilizaron con más frecuencia AP y Urgencias, mientras que la utilización de AE y Hospital de Día fue mayor en clases altas. Existen diferencias en salud y utilización de servicios sanitarios en mayores según clase social. Resulta necesario prestar atención a la salud percibida como predictor de la utilización de servicios sanitarios y revisar la accesibilidad-equidad de nuestros servicios.

Según algunos autores, las características de la población explicarían el 50\% de las variaciones de utilización, y factores del profesional y la organización el otro 50\% (Brugos, Guillen, Giménez, y Fernández, 2003). En cambio, otros autores atribuyen el $82 \%$ de la frecuentación a la edad y el estado de salud (Arnal, Amorin, y Castanc, 2001).

Algunas de las hipótesis pos las que se produce la hiperfrecuentación médica según Guerra y Anca (2007) son los factores relacionados con la necesidad de cuidados (percepción de necesidad física o psíquica o de apoyo emocional, tipo y duración de la enfermedad) son valorados por los participantes como «fundamentales» para explicar/ determinar su conducta, ya que consideran que son una amenaza grave (problemas cardiovasculares), o deterioran su calidad de vida (osteoarticulares) 0 sus interacciones sociales (problemas emocionales). A éstos se unen (necesarios pero no suficientes) factores facilitadores (conocimiento, accesibilidad a los servicios, experiencias previas, actuación y buena relación con los profesionales) y predisponentes (pensamientos sobre la salud, personalidad, nivel económico, edad, sexo, red social) para tomar la decisión de utilizar los servicios. Algunos de estos pueden actuar como facilitadores/barreras para la decisión de utilización y para mantenerla.

En el estudio de Parejo et al., (2015) se señaló que la hiperfrecuentación se relacionó con el sexo, la edad, el estado civil, el nivel académico, la estructura familiar, la existencia de enfermedad crónica, el consumo de ansiolíticos y antidepresivos, la solicitud de exámenes complementarios y derivaciones a otros especialistas, la proximidad al centro de salud y el grado de satisfacción con su médico de familia. En cuanto a las variables sociodemográficas, la mayoríade trabajos coinciden en señalar algunas características aso-ciadas a la hiperfrecuentación, como la edad avanzada, el sexo femenino, el bajo nivel económico y educacional, lajubilación, el desempleo o el hecho de vivir solo. Si atendemos a aspectos no sociodemográficos, los estudios son consistentes en afirmar que los hiperfrecuentadores presentan elevada frecuencia de enfermedades crónicas, físicas o psíquicas. En general, los datos publicados indican que entre los pacientes hiperutilizadores más del $50 \%$ tienen una enfermedad física y más de la mitad presentan algún tipo de malestar psicológico.

Los profesionales diferencian los motivos de consulta de los pacientes hiperfrecuentadores en 2 tal y como señalan Sandín-Vázquez, y Conde-Espejo (2010): por motivos de salud y por motivos no estrictamente médicos. Entre los motivos médicos, destacan pacientes crónicos con pluripatología (hipertensión, diabetes, cardiopatía, tumores o infartos), pacientes con procesos agudos que acuden por dolores inespecíficos y síntomas poco concluyentes (cefaleas, dolor de estómago 0 fibromialgia), pacientes con dolores relacionados con la edad (artrosis y dolores articulares), con problemas ginecológicos, con dolores musculares relacionados con el trabajo, y con enfermedad psicológica (ansiedad, estrés, mala adaptación, depresión o trastornos de personalidad). Entre los 


\section{HIPERFRECUENTACIÓN MÉDICA EN MAYORES Y SALUD}

motivos no estrictamente médicos, todos los profesionales coinciden en que habitualmente el hiperfrecuentador lleva asociada una problemática social o familiar, que lo hace acudir a la consulta de AP como un servicio de ayuda. Una de estas múltiples situaciones es la de pacientes con polipatología crónica y muy dependientes del sistema, mayores de 65 años, que acuden por los seguimientos que se realizan a su enfermedad y por problemas sintomáticos. Otra de las situaciones destacadas por los profesionales es la soledad. Según su opinión, las mujeres sin una red familiar o de apoyo son las que más tienen este problema. Los profesionales también hablan de problemas familiares, sociales y laborales que afectan a la población en general y se manifiestan en concreto en hipocondrías o situaciones de sobresaturación e insatisfacción. En el caso de la hipocondría, la percepción es que se trataría más de hombres y, en el segundo caso, se encontrarían más mujeres, algunas al cuidado de familiares, y en algunas de ellas se destaca el diagnóstico de fibromialgia.

Recientemente Fernández-Alonso et al., (2018) señalan que el hecho de ser mayor de 65 años, tomar 3 o más fármacos, presentar deterioro cognitivo y vivir en la comunidad se consideran factores predictores de ser HF también en AP.

\section{CONCLUSIONES}

El trabajo del médico de familia no es solo para ver la enfermedad y establecer un diagnóstico y un tratamiento farmacológico, sino también para ir más allá, como se ha mencionado por Doherty y Baird, 1983, citados en Riquelme-Heras, Gómez-Gómez, Gutiérrez-Herrera, Martínez-Lazcano, y Sierra-Ayala (2016) en la que el médico interviene en el cuarto nivel. En este nivel, el médico diagnostica la enfermedad física e identifica sentimientos de los pacientes y la dinámica familiar para implementar breves técnicas de intervención que mejoren la terapia del paciente o situación familiar.

Pensamos que una buena conclusión para este trabajo se basaría en lo señalado por SandínVázquez, y Conde-Espejo (2010), en la que según los profesionales de AP, la definición de un paciente como hiperfrecuentador no depende del número sino de la calidad de la visita. Las razones de consulta y las características de los pacientes son múltiples, por lo que no se puede determinar un perfil único. Asimismo, otra de las cuestiones en la que coinciden los diferentes estudios y que resaltan Sandín-Vázquez, y Conde-Espejo (2010), se refiere a la característica que aparece recurrentemente en los discursos es la de personas que demandan atención repetidamente para resolver problemas que el profesional no puede solucionar en la consulta de AP por ser más de salud psicosocial. Por lo que sería de gran ayuda agilizar la coordinación con recursos psicosociales para conseguir consultas de AP más eficientes y efectivas. Los mismos autores, concluyen de acuerdo con la mayoría de los estudios revisados, que para poder manejar el fenómeno de la hiperfrecuentación en AP (tan relacionado con los determinantes sociales de salud) y favorecer con esto la calidad asistencial, sería de gran ayuda agilizar la coordinación con recursos psicosociales para descargar a los profesionales de un trabajo que ni está en sus manos ni les es reconocido. Esto mejoraría la calidad de las consultas de AP y proporcionaría muchos pacientes (entre ellos los hiperfrecuentadores) una vía efectiva para la solución de sus problemas reales.

\section{REFERENCIAS}

Aguilar-Palacio, I., Carrera-Lasfuentes, P., Solsona, P., Sartolo, M. T. y Rabanaque, M. J. (2016). Utilización de servicios sanitarios en ancianos (España 2006-2012): influencia del nivel de salud y de la clase social. Atención Primaria, 48(4), 235-243.

Alpern, E. R., Clark A. E., Alessandrini E. A., Gorelick M. H., Kittick M., Stanley R. M.,... Chamberlain, J. M. (2014). Recurrent and high-frequency use of the emergency department by pediatric 
patients. Academic Emergency Medicine, 21(4), 365-73.

Arnal, J. M., Amorin, M. J., y Castanc, Y. (2001). Análisis cartográfico de la utilización de atención primaria en la Comunidad Autónoma de Aragón. Cuadernos de Gestión, 7, 73-84.

Baez, K., Aiarzaguena, J. M., Grandes, G., Pedrero, E., Aranguren, J., Retolaza, A. (1998). Understanding patient-initiated frequent attendance in primary care: a case-control study. British Journal of General Practice, 437, 1824-1827.

Bellón, J. A. (1995). Modelo explicativo de la hiperutilización en Atención Primaria [tesis doctoral]. Granada: Universidad de Granada.

Bellón, J., Rodríguez, A., Castillo, R., Gallardo, A., Caballero de las Olivas, M., y Moreno, I. (2004). Intervención sobre pacientes utilizadores de atención primaria: ensayo aleatorio controlado. Atención Primaria, 34(e1), 154.

Brugos, A.,Guillen, M., Giménez, C., Fernández, F. (2003). Modelos explicativos y predictivos de la carga asistencial médica: aplicación para el cálculo del cupo máximo en medicina de familia que permita un mínimo de diez minutos por consulta. Atención Primaria, 32, 23-9.

Cotonat, M. C., Bertolet, P. M., Ocaña, M. P., y Gatius, J. R. (2014). Análisis del perfil de los pacientes hiperfrecuentadores de Lleida (comparativa entre la Atención Primaria y la Atención Hospitalaria) y su relación con los factores sociales/psicosociales. Trabajo Social y Salud, 78, 512.

Dowrick, C. F., Bellón, J. A., y Gómez, M. J. (2000). Frequent attendance in Liverpool and Granada: the impact of depressive symptoms. British Journal of General Practice, 50, 361-365.

Fernández-Alonso, C., Aguilar-Mulet, J. M., Romero-Pareja, R., Rivas, A., Fuentes, M. E., GonzálezArmengol, J. G. (2018). Hiperfrecuentación en Atención Primaria e hiperfrecuentadores en Urgencias. Atención Primaria, 50, 222-227.

Fuertes, M. C., Álvarez, E., García, M. D., Fernández, M., Echarte, E., y Abad, J. (1994). Características del paciente hiperfrecuentador de las consultas de demanda de medicina general. Atención Primaria, 1(14), 809-14.

Guerra, J. A. y Anca, I. A. (2007). Motivos que condicionan el uso de los servicios sanitarios por parte de los pacientes hiperfrecuentadores: estudio con grupos focales. Atención Primaria, 39(7), 349-354.

Gill, D., y Sharp, M. (1999). Frequent consulters in general practice:A systematic review of studies of prevalence, associations andoutcome. Journal of Psychosomatic Research, 47, 15-30.

Llorente, S., López, T., García, L. J., Alonso, M., Alonso, P., Muñoz, P. (1996). Perfil del hiperfrecuentador en un centro de salud. Atención Primaria, 1(17), 100-106.

Luciano, J. V., Fernández, A., Pinto-Meza, A., Luján, L., Bellón, J. A.,García-Campayo, J. (2010). Frequent attendance in primary care: Com-parison and implications of different definitions. Brithis Jounal of General Practice, 60, 49-55.

Luciano, J. V. y Serrano, A. (2008). Los hiperfrecuentadores en atención primaria: perfil sociodemográfico, características clínicas y propuesta de una nueva definición. Atención Primaria, 40(12), 631-632.

Ortega, M. A., Roca, G., Iglesias, M., Jurado, J. M. (2004). Pacientes hiperfrecuentadores de un centro de atención primaria: características sociodemográficas, clínicas y de utilización de servicios. Atención Primaria, 33(2), 78-85.

Riquelme-Heras, H., Gómez-Gómez, C., Gutiérrez-Herrera, R., Martínez-Lazcano, F., y Sierra-Ayala, I. (2016). Criterios para identificar pacientes vulnerables en Atención Primaria. Revista Cubana de Medicina General Integral, 32(1), 18-27.

Rivera-Casares, F., Illana, A., Oltra, A., Narváez, M., Benlloch, C., Rovira, B. (2000). Características 


\section{HIPERFRECUENTACIÓN MÉDICA EN MAYORES Y SALUD}

de los pacientes que no utilizan las consultas de atención primaria. Gaceta Sanitaria, 14, 117121.

Rodríguez, C., Cebrià, J., Corbella, S., Segura, J., y Sobrequés, J. (2003). Rasgos de personalidad y malestar psíquico asociados a los pacientes hiperfrecuentadores de consultas de Atención Primaria. Revista de Medicina Familiar y Comunitaria, 13(3), 23-30.

Rubio, M. L., Adalid, C., Cordón, F., Solanas, P., Masó, D., y Fernández, D. (1998). Hiperfrecuentación en atención primaria: estudio de los factores psicosociales. Atención Primaria, 1(22), 627-630.

Sandín, B. (1999). El estrés psicosocial: concepto y consecuencias clínicas. Madrid: Klinik.

Sandín-Vázquez, M., y Conde-Espejo, P. (2010). Hiperfrecuentación: percepción de los profesionales de atención primaria sobre su definición, motivos, situaciones y la relación con los pacientes hiperfrecuentadores. Revista de Calidad Asistencial, 25(1), 21-27.

Sarría, A., Timoner, J., y Sandín, M. (2005). Frecuentación en Atención Primaria. Un replanteamiento conceptual. Atención Primaria, 36, 471-472.

Sihvonen, M., y Kekki, P. (1990). Unnecessary visits to health centres as perceived by the staff. Scandinavian Journal of Primary Health Care, 8(4), 233-9.

Vedsted, P., y Christensen, M. B. (2005). Frequent attenders in generalpractice care: A literature review with special reference to methodological considerations. Public Health, 119, 118-137.

Vidal, R., Cebria, J., Palma, C., Planells, G., Espinassa, J., Estafanell, A. (2010). El apoyo social percibido en pacientes hiperfrecuentadores de Atención Primaria. Butlletí, 28(3). Article 3. Recuperado de http://pub.bsalut.net/butlleti/vol28/iss3/3 